\title{
EXPANSÃO DA FRONTEIRA AGRÍCOLA: IMPACTO DAS POLÍTICAS DE DESENVOLVIMENTO REGIONAL NO CENTRO- OESTE BRASILEIRO
}

\author{
EXPANSION OF AGRICULTURAL FRONTIER: IMPACT OF \\ POLICIES ON REGIONAL DEVELOPMENT IN THE CENTRE-WEST \\ OF BRAZIL
}

\author{
Giuliana Mendonça de Farias ${ }^{1}$ \\ Carlos Otávio Zamberlan ${ }^{2}$
}

\begin{abstract}
RESUMO
O processo de desenvolvimento da região Centro-Oeste teve impulso por meio de políticas e incentivos do Governo Vargas, entretanto, foi a partir dos anos 70, pelo II Plano Nacional de Desenvolvimento (PND), em especial o Programa de Desenvolvimento dos Cerrados (POLOCENTRO), que a estrutura produtiva da região foi alterada. Esse programa foi fundamental para inserir o cerrado nas áreas produtivas, por meio de seu aproveitamento em escala empresarial, e consequentemente promover o desenvolvimento da região. Este trabalho objetiva analisar os impactos que o POLOCENTRO causou na região Centro-Oeste. Para tanto, utilizou-se como metodologia uma análise de Taxa Geométrica de Crescimento aplicada a variáveis relacionadas ao fomento da moderna agricultura, que é o objetivo do programa. Concluiu-se que o POLOCENTRO, aliado às condições ambientais dos cerrados, exerceu grande impacto na modernização da agricultura, mas não houve preocupação com questões sociais, gerando concentração no uso de terras.
\end{abstract}

Palavras-chave: II PND; POLOCENTRO; Modernização.

\begin{abstract}
The development of the Brazilian center-west region was stimulated through policies and investments promoted by the administration of Getulio Vargas. However, the productive structure of this region was altered by the Second National Program of Development (PND), mainly, by Cerrados Development Program (POLOCENTRO), from the 1970s on. This program was fundamental to insert cerrado region into the group of productive areas through its utilization in an enterprise scale and, consequently, promote its development. This study aims at analyzing the impact of POLOCENTRO on the center-west region. To accomplish such purpose, an analysis of Geometric Rate of Growth applied to variables related to the promotion of modern agriculture, which is the objective of the program - was used. The results suggest that POLOCENTRO, allied to environmental conditions of cerrado, exerted great impact on the modernization of agriculture. However, there was no concern with social issues causing concentration in land use.
\end{abstract}

Keywords: Second PND; Polocentro; Modernization.

\footnotetext{
${ }^{1}$ Doutora em Economia pela Universidade Federal do Rio Grande do Sul (UFRGS) e professora titular do Curso de Administração da Universidade Estadual de Mato Grosso do Sul (UEMS), Maracaju, e do curso de Turismo da UEMS, Campo Grande.

${ }^{2}$ Doutor em Economia pela Universidade Federal do Rio Grande do Sul (UFRGS), Mestre e Bacharel em Administração pela Universidade Federal de Santa Maria (UFSM); professor da Universidade Estadual de Mato Grosso do Sul (UEMS).
} 


\section{Introdução}

O processo de desenvolvimento da região Centro-Oeste teve impulso na década de 1930, no governo Vargas, com a criação de políticas estatais de colonização, interiorização e integração econômica. Entretanto, a intervenção mais efetiva neste sentido deu-se durante o governo militar a partir da década de 1970, principalmente com o II Plano Nacional de Desenvolvimento - II PND (1975-1979), que tinha como principal objetivo a expansão da fronteira agrícola no país.

Na década de 1970 ainda era predominante a visão de que o cerrado não oferecia potencial para exploração produtiva. Entretanto, os avanços da modernização agrícola, impulsionados pelas ações e políticas do governo, contribuíram substancialmente para uma nova configuração da estrutura produtiva do Centro-Oeste, gerando assim o seu desenvolvimento.

O objetivo deste artigo é analisar os impactos na região Centro-Oeste, das políticas de desenvolvimento regional implantadas na década de 1970 durante o II PND, com enfoque no Programa de Desenvolvimento dos Cerrados (POLOCENTRO), que tinha como objetivo expandir a fronteira agrícola do país por meio da modernização agrícola voltada para o Cerrado.

Inicialmente, apresenta-se em linhas gerais o processo de ocupação e formação do Centro-Oeste, por este ter sido afetado diretamente pelas políticas e ações do governo; a seguir, aborda-se o POLOCENTRO com base no referencial teórico de polos de desenvolvimento. Em seguida, apresenta-se a metodologia utilizada para a análise dos impactos desse programa na região, decorrentes da expansão da agricultura comercial.

\section{Ocupação e formação do Centro-Oeste}

A região Centro-Oeste é composta pelos Estados de Goiás, Mato Grosso, Mato Grosso do Sul e Distrito Federal. De acordo com Oliveira (2002), a ocupação do Centro-Oeste teve início no final do século XVII, a partir dos primeiros fluxos migratórios, atraídos pela descoberta de ouro aluvial na região. Com o esgotamento das jazidas no final do século XVIII, iniciou-se uma fase de estagnação econômica, que deixou a região praticamente desabitada.

Somente na década de 1930 houve incentivo oficial para o povoamento do CentroOeste através da chamada "Marcha para o Oeste", criada pelo governo Getúlio Vargas para incentivar o progresso e a ocupação efetiva do Centro-Oeste. Um dos objetivos do governo Vargas era promover a integração do mercado nacional através da interiorização da economia, sendo a região Centro-Oeste contemplada com dois projetos de colonização: a Colônia Agrícola Nacional de Goiás na cidade de Ceres/GO e a Colônia Pecuária Nacional de Dourados no atual Mato Grosso do Sul (ALMEIDA, 2006).

A região, no final dos anos de 1930, ainda se encontrava bastante despovoada, apresentando uma infra-estrutura deficiente que dificultava sua ligação com os grandes centros econômicos do país. O processo de ocupação da região Centro-Oeste foi consolidado com a criação de dois grandes núcleos urbanos, Goiânia e Brasília, acentuando o investimento em infra-estrutura (TEIXEIRA; HESPANHOL, 2006). 
A inauguração de Goiânia como nova capital de Goiás, em 1937, representou um forte estímulo à migração para a região. Entretanto, foi a construção de Brasília, que fazia parte do Plano de Metas (1956-1961) do governo Juscelino Kubitscheck, que acelerou o processo de migração para a região, através de investimento em infra-estrutura e da implantação de importante malha rodoviária ligando a região com os principais centros econômicos nacionais (ALMEIDA, 2006).

O esgotamento de terras disponíveis para a ocupação da agropecuária nas regiões Sul e Sudeste do país, bem como a necessidade de aumento da produtividade agrícola, gerou a necessidade de direcionar a produção para novas áreas e a conseqüente expansão agrícola (OLIVEIRA, 2002). A falta de programas específicos e incentivos, até a década de 1960, bloqueava, de certa maneira, o desenvolvimento agrícola na região.

Segundo Oliveira (2002), a partir dos anos 1960, as políticas de integração e desenvolvimento regional sofreram mudanças substanciais, adotou-se uma nova orientação de desenvolvimento, através de políticas e programas de desenvolvimento regional que visavam o aproveitamento dos cerrados e a abertura de novas terras para a exploração agropecuária. Neste contexto, a região Centro-Oeste tornou-se estratégica, tanto pela sua posição geográfica quanto pelas suas características físico-ambientais, que propiciavam a expansão da produção agrícola nos padrões da nova agricultura moderna.

A partir do final dos anos 60 inaugura-se uma nova fase na região Centro-Oeste, caracterizada por um fluxo imigratório menos intenso, porém mais qualificado, composto por paulistas, paranaenses e gaúchos, em geral possuidores de capital e/ou experiência na atividade agrícola (GUIMARÃES; LEME, 1998). Assim, a região Centro-Oeste, a partir da década de 1970, ganha novas funções na economia brasileira, o que impulsiona o seu desenvolvimento.

Durante o governo militar, nas décadas de 1960 e 1970, foram implantados diversos programas de caráter nacional e regional que impactaram a região Centro-Oeste (ALMEIDA, 2006). Esse período é fundamental para entender a situação atual da região, pois corresponde ao momento de maior intervenção do governo federal, que através de políticas e programas direcionados para o Centro-Oeste, acarretaram mudanças importantes na estrutura produtiva da região.

O Centro-Oeste teve Mato Grosso e Goiás como duas unidades fundamentais no progresso sócio-econômico regional desde os tempos coloniais, tanto que nesta etapa de fortalecimento e expansão do mercado interno, Mato Grosso do Sul e o Distrito Federal desmembraram-se devido à dinâmica política e econômica que envolvia esses dois estados.

A ocupação e formação da região Centro-Oeste, que devido a sua posição privilegiada de proximidade dos grandes centros consumidores, tem suas bases fincadas nas políticas e ações do governo que se propunha a promover o desenvolvimento regional. De acordo com Abreu (2004), a justificativa para as ações promovidas pelo governo era acabar com as desigualdades regionais e redistribuir de maneira mais justa a renda nacional produzida.

\section{Programa de desenvolvimento dos Cerrados - POLOCENTRO}

Durante o governo militar, as políticas de desenvolvimento regional criadas pelo II Plano Nacional de Desenvolvimento (PND) adotaram um modelo de planejamento voltado para regiões específicas do país. Essas políticas fundamentaram-se, de maneira geral, nos conceitos de polos de crescimento e na dinâmica de atração de investimentos, que, segundo 
Tolosa (1972), foram desenvolvidos por François Perroux e seus colaboradores como uma tentativa de resposta aos problemas criados pelos desequilíbrios regionais.

Para coordenar o desenvolvimento da região Centro-Oeste, foi criada em 1967, ainda durante o I PND, a Superintendência do Desenvolvimento do Centro-Oeste (SUDECO), que tinha como principais funções realizar pesquisas visando conhecer o potencial econômico da região e definir áreas apropriadas para o desenvolvimento planejado, por meio da fixação de polos de crescimento capazes de desenvolver áreas vizinhas (OLIVEIRA, 2002).

Conforme aponta Tolosa (1972), o espaço ou região do programa é definido em termos de unidade de decisão, e consiste, portanto, em um espaço ou região sob a mesma autoridade ou centro de decisão. Os polos de desenvolvimento podem surgir como resultado da atuação de forças livres de mercado, denominados polos naturais, ou servir como instrumento da política econômica governamental, a fim de atingir um conjunto de objetivos nacionais de desenvolvimento regional, chamados de polos planejados.

Ainda de acordo com Tolosa (1972), especialmente nas economias em desenvolvimento, o estabelecimento de uma região programa pode ter como principal objetivo a criação de regiões polarizadas, que geralmente envolvem a integração de regiões desabitadas no espaço econômico nacional por meio de mudanças estruturais.

Conforme descreve Perroux (1967), o crescimento e o desenvolvimento de um conjunto de territórios e de populações serão obtidos a partir da organização consciente do meio de propagação dos efeitos do polo de desenvolvimento. Em outras palavras, são órgãos de interesse geral que transformam o crescimento de uma região ou atividade em crescimento de uma nação em desenvolvimento.

O Programa de Desenvolvimento dos Cerrados (POLOCENTRO), formulado pela SUDECO a partir das diretrizes do II PND, tinha como objetivo incorporar racionalmente, ou seja, em bases empresariais, a região Centro-Oeste ao espaço econômico nacional, estimulando os produtores rurais a adotar inovações tecnológicas, visando otimizar os resultados econômicos dos seus empreendimentos (PIRES, 2000).

De acordo com Diniz (2006), até meados dos anos 1970, notam-se políticas de desenvolvimento regionais com ênfase na correção das disparidades inter-regionais. Essas teorias postulavam que o desenvolvimento regional não era garantido automaticamente pelas forças de mercado, sendo necessária a intervenção estatal para que os desequilíbrios regionais fossem superados.

No documento síntese do II PND, a região Centro-Oeste desempenhava papel importante na estratégia de crescimento nacional, assumindo as funções de produzir alimentos e matérias-primas voltadas para a exportação e absorvendo as correntes migratórias de mão de obra (OLIVEIRA, 2002). As diretrizes para a atuação governamental nesse período consistiam em concentrar esforços nas áreas prioritárias, expandindo a fronteira agrícola e ampliando a infra-estrutura de transporte.

Consoante Abreu (2004), o POLOCENTRO foi um dos programas desenvolvidos dentro da estratégia de incorporar a região Centro-Oeste dentre as áreas que deveriam receber investimentos para promover o que a SUDECO chamou de "fronteira econômica", substituindo a agricultura de subsistência e a policultura em favor da monocultura comercial, visando a ampliação do mercado nacional e de exportação.

O principal instrumento de incentivo do POLOCENTRO foi o crédito rural subsidiado, que era amplo e extremamente atrativo, estendido aos que quisessem investir na 
exploração empresarial nas áreas selecionadas (OLIVEIRA, 2002). De acordo com Tolosa (1972), o número total de polos pode ser fixado em função do volume de recursos financeiros destinados à implantação.

Dentro da concepção de polos de crescimento que orientou os programas de desenvolvimento do governo federal entre 1975 e 1979, o POLOCENTRO selecionou áreas dotadas de infra-estrutura e com potencial para a expansão agrícola comercial. Segundo Almeida (2006), os municípios estimulados por esse programa foram: Campo Grande, Três Lagoas e Bodoquena no Mato Grosso do Sul; Xavantina e Parecis no Mato Grosso; e Paraná, Pirineus, Piranhas e Rio Verde em Goiás.

No que se refere ao desenvolvimento tecnológico, especial atenção deve ser dada à criação, em 1973, da Empresa Brasileira de Pesquisa Agropecuária (EMBRAPA), responsável pela geração de tecnologias aptas para as características específicas do cerrado. No âmbito estadual essa responsabilidade foi exercida pelas empresas de pesquisa, como a Empresa de Pesquisa Agropecuária do Estado de Minas Gerais (EPAMIG), Empresa Agropecuária do Estado de Goiás (EMGOPA) e Empresa Agropecuária do Estado de Mato Grosso (EMPA-MT) (SILVA, 2000). A EMBRAPA procurou contribuir tanto para o aumento da produtividade quanto para a expansão da fronteira agrícola pela incorporação de novas áreas ao processo produtivo.

Outro fator importante, ao lado do desenvolvimento tecnológico, foi o crédito agrícola, que teve papel crucial nesse programa de desenvolvimento do cerrado. De acordo com Abreu (2001), o maior suporte do POLOCENTRO era o crédito rural subsidiado associado à pesquisa agropecuária, que dispunha de linhas especiais de crédito que cobriam todas as etapas da produção, desde a elaboração do projeto para a propriedade rural, compra de implementos agrícolas, plantio, colheita e construção de armazéns e silos.

O POLOCENTRO, assim como outros programas, consistia na conjugação de subprogramas que estavam dentro de linhas que consistiam em: pesquisa agropecuária; promoção e extensão agropecuária; estradas vicinais; eletrificação rural; implantação de sistemas de beneficiamento, armazenagem e transporte de produtos agrícolas; e estímulos à produção e comercialização regional de calcário e outros insumos agrícolas (ABREU, 2001).

De acordo com Tolosa (1972), de maneira geral, os instrumentos comumente utilizados na programação de um sistema de polos planejados, com maior ou menor intensidade e de um modo coordenado, podem ser classificados em:

a) Investimentos do governo em infra-estrutura visando criar condições ou induzir a implantação de atividades produtivas, e em particular de investimentos privados;

b) Políticas de localização em que o governo procura influenciar, direta ou indiretamente, os padrões locacionais do setor privado em favor das áreas consideradas prioritárias;

c) Mobilidade de fatores, particularmente da mão de obra, por meio do controle e reorientação dos fluxos migratórios;

d) Promoção, ou seja, trabalho de divulgação a investidores em potencial das oportunidades de investimento existente nos polos.

Em economias em desenvolvimento, o governo geralmente comanda maciços programas de infra-estrutura, visando criar condições favoráveis à concentração de atividades privadas nos polos selecionados (TOLOSA, 1972). De acordo com Almeida (2006), esse período foi fundamental para a formação da estrutura produtiva atual, pois o capital privado 
encontra na infra-estrutura, nos incentivos fiscais e no crédito estatal as condições de investimento na região expandida a partir das características de fronteira agrícola.

As experiências direcionadas para a redução de desequilíbrios regionais podem ser consideradas relativamente bem-sucedidas em termos de crescimento econômico em algumas regiões, ainda que tenham permanecido elevados níveis de concentração regional da produção e persistentes desigualdades sociais (DINIZ, 2006). Dentre os programas voltados para a região, o POLOCENTRO foi de fundamental importância, porque materializou outra forma de intervenção do governo para viabilizar o desenvolvimento agrícola no cerrado.

\section{Metodologia}

Neste trabalho, para analisar os impactos do POLOCENTRO na região Centro-Oeste, foram utilizadas as seguintes variáveis: população total, extensão da rede rodoviária e população por situação do domicílio, disponibilizados pelo IBGE; total de tratores existentes nos estabelecimentos agropecuários, quantidade em hectares de terras irrigadas e utilização da terra para a agricultura temporária e permanente, disponibilizados pelo IEPADATA.

Optou-se por essas variáveis em função do objetivo principal do programa, que é fomentar a agricultura moderna na região por meio de amplos créditos subsidiados para introdução e transferência de tecnologias consideradas modernas. Para melhor comparar e analisar as variáveis de estudo, utilizou-se as Taxas Geométricas de Crescimento (TGC) anuais, calculadas com o auxílio do Excel 2003, através da fórmula abaixo:

$$
\begin{aligned}
& \text { TGC }=\left(\frac{y}{x}\right)^{1 / n}-1 \text { onde, } \\
& \mathrm{y}=\text { último dado do período analisado. } \\
& \mathrm{x}=\text { primeiro dado do período analisado. } \\
& \mathrm{n}=\text { número de período do intervalo analisado em anos. }
\end{aligned}
$$

Utilizou-se a TGC para poder analisar o crescimento existente na região para cada variável analisada. A TGC permite verificar se o crescimento indicado pelos números absolutos ocorrem com as suas taxas, possibilitando uma comparação entre os períodos escolhidos para compor o estudo.

\section{Impactos do POLOCENTRO na região Centro-Oeste}

As políticas de desenvolvimento regional implantadas pelo governo federal desde os anos 30 foram fundamentais para o processo de ocupação e desenvolvimento econômico do Centro-Oeste. Posteriormente, a construção de Brasília exerceu um papel atrativo de contingentes populacionais oriundos de outras regiões, entretanto, o ritmo de ocupação na região acelerou, a partir da década de 1970, durante os governos militares com a implantação de planos estratégicos de desenvolvimento.

O II PND apresentava em suas diretrizes como meta mais importante a expansão da fronteira agrícola por meio do aproveitamento do cerrado, que até então era tido como inaproveitável, e a abertura de novas terras para a produção agrícola. Desse modo, observa-se 
que o POLOCENTRO foi fundamental para impulsionar a inserção do cerrado nas áreas produtivas e, consequentemente, o desenvolvimento agrícola da região Centro-Oeste.

Assim, os impactos decorrentes desse programa devem ser analisados sob a ótica da expansão da fronteira agrícola e da introdução de modernas tecnologias de produção para o cerrado, favorecendo o seu aproveitamento em escala empresarial. Esses fatores foram decisivos para a mudança da estrutura produtiva da região, tornando-a uma área de produção agrícola moderna.

A expansão econômica do Centro-Oeste, estimulada pelos programas de desenvolvimento regional, foi acompanhada por grandes fluxos migratórios, gerando um aumento nas taxas de crescimento populacional, principalmente durante o período de 1950 a 1970, como pode ser observado na Tabela 1:

Tabela 1 - Taxa Geométrica de Crescimento da população na região

\begin{tabular}{|c|c|c|c|c|c|c|c|c|c|c|}
\hline \multirow[b]{2}{*}{ UF } & \multicolumn{2}{|c|}{$\underline{40 / 50}$} & \multicolumn{2}{|c|}{$\underline{50 / 60}$} & \multicolumn{2}{|c|}{$60 / 70$} & \multicolumn{2}{|c|}{$7 \underline{70 / 80}$} & \multicolumn{2}{|c|}{$\underline{80 / 91}$} \\
\hline & $\overline{\text { Absoluto }}$ & $\%$ & $\overline{\text { Absoluto }}$ & $\%$ & $\overline{\text { Absoluto }}$ & $\%$ & $\overline{\text { Absoluto }}$ & $\%$ & Absoluto & $\%$ \\
\hline MS & 0,026306 & 2,63 & 0,064794 & 6,48 & - & - & 0,043936 & 4,39 & 0,024121 & 2,41 \\
\hline MT & 0,009416 & 0,94 & 0,045118 & 4,51 & 0,170575 & 17,06 & $-0,033240$ & $-3,32$ & 0,053816 & 5,38 \\
\hline GO & 0,043362 & 4,34 & 0,048702 & 4,87 & 0,060922 & 6,09 & 0,027673 & 2,77 & 0,003670 & 0,37 \\
\hline $\mathrm{DF}^{*}$ & - & - & - & - & 0,142582 & 14,26 & 0,081526 & 8,15 & 0,028377 & 2,84 \\
\hline $\mathrm{CO}$ & $\mathbf{0 , 0 3 4 3 5 7}$ & $\overline{3,44}$ & $\overline{0,057390}$ & $\overline{5,74}$ & 0,065946 & 6,59 & $\overline{0,040514}$ & $\overline{4,05}$ & $\overline{0,020448}$ & 2,04 \\
\hline
\end{tabular}

Fonte: Elaborado pelos autores.

Além do Distrito Federal, devido à construção de Brasília, pode-se considerar que o Mato Grosso apresentou elevada taxa de crescimento populacional durante o período de 1970 a 1980, em parte motivada pela expansão da fronteira agrícola e em parte por causa do baixo contingente populacional da região que acarretava a atração de migrantes.

Em consequência da grande atração populacional, atraiu-se para a região uma gama de investimentos em infra-estrutura e a implantação de importante malha rodoviária, ligando a região com os principais centros econômicos nacionais.

A Tabela 2, abaixo, demonstra que o crescimento da rede rodoviária na região CentroOeste, em função dos fatores anteriormente descritos, se mantém acima do crescimento da rede rodoviária brasileira.

Tabela 2 - Taxa Geométrica de Crescimento da extensão da rede rodoviária da região Centro-Oeste

\begin{tabular}{ccccccccccc}
\hline & \multicolumn{3}{c}{$\mathbf{4 3 / 5 5}$} & \multicolumn{5}{c}{$\mathbf{5 5 / 6 4}$} & $\mathbf{6 4 / 7 1}$ & \multicolumn{2}{c}{$\mathbf{7 1 / 8 1}$} & \multicolumn{2}{c}{$\mathbf{8 1 / 9 1}$} \\
\cline { 2 - 11 } UF & Absoluto & \% & Absoluto & \% & Absoluto & \% & Absoluto & \% & Absoluto & \% \\
\hline CO & 0,012230 & 1,22 & 0,040638 & 4,06 & 0,135901 & 13,59 & 0,034340 & 3,43 & 0,015716 & 1,57 \\
\hline Brasil & 0,043214 & 4,32 & 0,019816 & 1,98 & 0,127772 & 12,78 & 0,009619 & 0,96 & 0,007152 & 0,72 \\
\hline
\end{tabular}

Fonte: Elaborado pelos autores.

O crédito rural subsidiado disponibilizado através do POLOCENTRO propiciou a introdução de tecnologias modernas que incorporaram o cerrado à produção agrícola 
moderna, beneficiando assim os agricultores da região a partir da década de 1970, conforme pode ser observado através do crescimento do uso de tratores na Tabela 3 e de irrigação na Tabela 4.

Tabela 3 - Taxa Geométrica de Crescimento da utilização de tratores na região

\begin{tabular}{|c|c|c|c|c|c|c|c|c|}
\hline \multirow[b]{2}{*}{ UF } & \multicolumn{2}{|c|}{$70 / 75$} & \multicolumn{2}{|c|}{$75 / 80$} & \multicolumn{2}{|c|}{$80 / 85$} & \multicolumn{2}{|c|}{$85 / 95$} \\
\hline & Absoluto & $\%$ & Absoluto & $\%$ & Absoluto & $\%$ & Absoluto & $\%$ \\
\hline $\mathrm{MS}$ & 0,121097 & 12,11 & 0,259903 & 25,99 & 0,070935 & 7,09 & 0,007320 & 0,73 \\
\hline MT & 0,183503 & 18,35 & 0,134332 & 13,43 & 0,064254 & 6,43 & 0,025877 & 2,59 \\
\hline $\mathrm{GO}$ & 0,265556 & 26,56 & 0,135110 & 13,51 & 0,060547 & 6,05 & 0,015903 & 1,59 \\
\hline DF & 0,345207 & 34,52 & 0,333774 & 33,38 & 0,118553 & 11,86 & 0,053040 & 5,30 \\
\hline $\mathrm{CO}$ & 0,225999 & 22,60 & 0,162091 & 16,21 & 0,073941 & 7,39 & 0,028923 & 2,89 \\
\hline
\end{tabular}

Fonte: Elaborado pelos autores.

Tabela 4 - Taxa Geométrica de Crescimento de terras irrigadas na região

\begin{tabular}{|c|c|c|c|c|c|c|c|c|}
\hline \multirow[b]{2}{*}{$\mathbf{U F}$} & \multicolumn{2}{|c|}{$60 / 70$} & \multicolumn{2}{|c|}{$70 / 75$} & \multicolumn{2}{|c|}{$75 / 80$} & \multicolumn{2}{|c|}{$\underline{80 / 85}$} \\
\hline & Absoluto & $\%$ & Absoluto & $\%$ & Absoluto & $\%$ & Absoluto & $\%$ \\
\hline MS & 0,267000 & 26,70 & 0,126251 & 12,63 & 0,180607 & 18,06 & 0,029680 & 2,97 \\
\hline MT & 0,182255 & 18,23 & 0,166307 & 16,63 & 0,204196 & 20,42 & $-0,018810$ & $-1,88$ \\
\hline GO & 0,230395 & 23,04 & 0,328401 & 32,84 & $-0,069723$ & $-6,97$ & 0,093893 & 9,39 \\
\hline $\mathrm{DF}$ & 0,513213 & 51,32 & $-0,210420$ & $-21,04$ & 0,299933 & 29,99 & 0,246270 & 24,63 \\
\hline $\mathrm{CO}$ & 0,242527 & 24,25 & $\mathbf{0 , 1 9 8 3 9 7}$ & 19,84 & $\mathbf{0 , 0 5 8 7 5 5}$ & 5,88 & 0,060118 & 6,01 \\
\hline
\end{tabular}

Fonte: Elaborado pelos autores.

Na Tabela 5, pode-se visualizar a maneira como a expansão agrícola na região CentroOeste foi paulatinamente ocupando o cerrado.

Tabela 5 - Utilização da terra na região Centro-Oeste

\begin{tabular}{|c|c|c|c|c|c|c|c|c|}
\hline \multirow{3}{*}{$\mathbf{U F}$} & \multicolumn{2}{|l|}{ 60/70 } & \multicolumn{2}{|l|}{ 70/75 } & \multicolumn{2}{|c|}{ 75/80 } & \multicolumn{2}{|c|}{ 80/85 } \\
\hline & \multicolumn{8}{|c|}{ Lavoura permanente } \\
\hline & Absoluto & $\%$ & Absoluto & $\%$ & Absoluto & $\%$ & Absoluto & $\%$ \\
\hline $\mathrm{MS}$ & 0,169823 & 16,98 & $-0,036698$ & $-3,67$ & 0,195185 & 19,52 & $-0,011341$ & $-1,13$ \\
\hline MT & 0,491581 & 49,16 & $-0,050143$ & $-5,01$ & 0,044503 & 4,45 & 0,012962 & 1,30 \\
\hline GO & $-0,011628$ & $-1,16$ & 0,110757 & 11,08 & $-0,044386$ & $-4,44$ & $-0,115093$ & $-11,51$ \\
\hline DF & 0,020622 & 2,06 & 0,142657 & 14,27 & 0,252120 & 25,21 & 0,010272 & 1,03 \\
\hline $\mathrm{CO}$ & 0,071329 & 7,13 & 0,047120 & 4,71 & 0,094706 & 9,47 & $-0,011268$ & $-1,13$ \\
\hline \multirow{3}{*}{$\mathbf{U F}$} & $60 / 70$ & & $\underline{70 / 75}$ & & $\underline{75 / 80}$ & & $\mathrm{80} / 8$ & \\
\hline & \multicolumn{8}{|c|}{ Lavoura temporária } \\
\hline & Absoluto & $\%$ & Absoluto & $\%$ & Absoluto & $\%$ & Absoluto & $\%$ \\
\hline MS & 0,123793 & 12,38 & 0,011317 & 1,13 & 0,405934 & 40,59 & 0,091921 & 9,19 \\
\hline MT & 0,060492 & 6,05 & 0,092828 & 9,28 & 0,037205 & 3,72 & 0,018342 & 1,83 \\
\hline GO & 0,071183 & 7,12 & 0,217449 & 21,74 & 0,056297 & 5,63 & 0,033534 & 3,35 \\
\hline $\mathrm{DF}$ & 0,118638 & 11,86 & 0,137391 & 13,74 & 0,253973 & 25,40 & 0,069612 & 6,96 \\
\hline $\mathrm{CO}$ & 0,374106 & 37,41 & 0,458984 & 45,90 & 0,753409 & 75,34 & 0,036982 & 3,70 \\
\hline
\end{tabular}

Fonte: Elaborado pelos autores. 
Por não encontrarem apoio de origem governamental, as culturas tradicionais foram perdendo espaço para a produção das culturas de exportação, desencadeando uma mudança no perfil regional. Essas mudanças na estrutura produtiva da região provocaram também mudanças nas relações de trabalho, em que a mão de obra temporária passou a predominar.

Apesar de o POLOCENTRO ter sido um programa voltado para a abertura de fronteira agrícola, as políticas favoreceram os grandes e médios produtores em detrimento dos pequenos. Do ponto de vista da estrutura fundiária, ocorreu redução das pequenas propriedades, pois as exigências para adequar-se aos novos padrões agrícolas geraram forte segregação, sendo responsável pela expulsão de muitos agricultores do campo e liberação de grande quantidade de pessoas que exerciam atividades que passaram a ser executadas por máquinas.

Em consequência do tipo de produção agrícola implantada na região, monocultora e intensiva em capital, portanto pouco geradora de empregos diretos, pois essas tecnologias podem ser utilizadas por poucas pessoas em grandes extensões de terras, houve intenso êxodo rural, principalmente no Mato Grosso e Mato Grosso do Sul. Na Tabela 6, a seguir, está demonstrado o percentual de pessoas na área urbana e rural da região:

Tabela 6 - População do Centro-Oeste por situação do domicílio (percentual)

\begin{tabular}{llrrrr}
\hline $\begin{array}{l}\text { Estados } \\
\text { Região }\end{array}$ & $\begin{array}{l}\text { Situação de } \\
\text { domicílio }\end{array}$ & $\mathbf{1 9 7 0}$ & $\mathbf{1 9 8 0}$ & $\mathbf{1 9 9 1}$ & $\mathbf{2 0 0 0}$ \\
\hline MS & Urbana & - & 67,11 & 79,45 & 84,08 \\
& Rural & - & 32,89 & 20,55 & 15,92 \\
\hline MT & Urbana & 42,82 & 57,52 & 73,26 & 79,35 \\
& Rural & 57,18 & 42,48 & 26,74 & 20,65 \\
\hline GO & Urbana & 42,17 & 62,2 & 80,81 & 87,88 \\
& Rural & 57,83 & 37,8 & 19,9 & 12,12 \\
\hline DF & Urbana & 96 & 96,78 & 94,68 & 95,66 \\
& Rural & 4 & 3,22 & 5,32 & 4,34 \\
\hline CO & Urbana & $\mathbf{4 8 , 0 8}$ & $\mathbf{6 7 , 7 8}$ & $\mathbf{8 1 , 2 8}$ & $\mathbf{8 6 , 7 3}$ \\
& Rural & $\mathbf{5 1 , 9 2}$ & $\mathbf{3 2 , 2 2}$ & $\mathbf{1 8 , 7 2}$ & $\mathbf{1 3 , 2 7}$ \\
\hline
\end{tabular}

Fonte: IBGE.

O crédito altamente subsidiado para implantação da agricultura comercial na região alterou sua base produtiva, aprofundando a concentração de terra em grandes propriedades, que aliadas à produção de monoculturas voltadas à exportação e intensiva em capital, não foram capazes de absorver o grande contingente populacional pela baixa geração de emprego, o que acaba aprofundando as desigualdades regionais.

\section{Conclusão}

A região Centro-Oeste sofreu grandes modificações a partir de meados do século XX, principalmente pela sua posição privilegiada de proximidade dos grandes centros consumidores, por meio de políticas e incentivos do governo federal no processo de reocupação nacional e no interesse pelo desenvolvimento da economia brasileira por meio do aumento e da modernização da produção agrícola.

Esse processo de modernização agrícola desencadeado a partir da década de 1950 atingiu a região Centro-Oeste de maneira efetiva somente na década de 1970, pelas estratégias 
dos planos de desenvolvimento regional, em especial o POLOCENTRO, intensificando o fluxo migratório oriundo de outras regiões.

As condições ambientais e a presença de extensas chapadas das áreas do Centro-Oeste, aliadas à atuação do governo no desenvolvimento de pesquisas e projetos de infra-estrutura, contribuíram para a utilização de sistemas de cultivo intensivo na região. Desse modo, a região Centro-Oeste incorpora as transformações ocorridas no processo produtivo a partir da década de 1970, apresentando grande melhoria nas formas produtivas, como maior mecanização e uso de implementos agrícolas.

Pode-se afirmar, portanto, que a década de 1970 foi marcada basicamente pela modernização da produção agrícola, largamente favorecida por programas e incentivos públicos. A região Centro-Oeste passou então a desempenhar papel mais relevante na economia nacional. Entretanto, o caráter produtivista do POLOCENTRO, concentrado na produção de produtos agrícolas exportáveis, destaca a ausência de preocupação com os aspectos sociais, como a concentração de renda, o êxodo rural e o desemprego.

\section{REFERÊNCIAS}

ABREU, Silvana de. Planejamento governamental: a SUDECO no espaço mato-grossense. Contexto, propósitos e contradições. 2001. 328 f. Tese (Doutorado em Geografia) Universidade de São Paulo, São Paulo, 2001.

. O Mato Grosso do Sul no contexto das políticas regionais de desenvolvimento. In: CONGRESSO BRASILEIRO DE GEÓGRAFOS, 6., 2004, Goiânia. Anais... Goiânia: AGB, 2004.

ALMEIDA, Giovanna S.; VIEIRA JUNIOR, Pedro A.; RAMOS, Pedro. Os programas de desenvolvimento econômico do centro-oeste brasileiro e suas consequências: anos 60 e 70. In: CONGRESO DE LA ASOCIACIÓN LATINOAMERICANA DE SOCIOLOGÍA RURAL, 7., 2006, Quito. Anais... Quito: 2006.

DINIZ, Clélio C.; CROCCO, Marco (Org.). Economia regional e urbana: contribuições teóricas recentes. Belo Horizonte: Editora UFMG, 2006.

GUIMARÃES, Eduardo N.; LEME, Heládio José de Campos. Caracterização histórica e configuração espacial da estrutura produtiva do Centro-Oeste. Textos Nepo, Campinas, n. 33, p. 21-65, 1998.

OLIVEIRA, Aparecida A. Análise dos impactos das políticas de desenvolvimento regional na Bacia do Alto Paraguai. Ensaios e Ciências, Campo Grande, v. 6, n. 3, p. 13-37, 2002.

PERROUX, François. A economia do século XXI. Lisboa: Herder, 1967.

PIRES, Mauro O. Programas agrícolas na ocupação do cerrado. Sociedade e Cultura, v. 3, n. 1 e 2, p. 111-131, 2000.

SILVA, Lílian L. O papel do Estado no processo de ocupação das áreas de cerrado entre as décadas de 60 e 80. Caminhos de Geografia, Uberlândia, v. 1, n. 2, p. 24-36, 2000.

TEIXEIRA, Jodenir C.; HESPANHOL, Antônio N. A região Centro-Oeste no contexto das mudanças agrícolas ocorridas no período pós-1960. Revista Eletrônica da Associação dos Geógrafos Brasileiros, Seção Três Lagoas/MS, v. 1, n. 3, p. 52-66, 2006. 
TOLOSA, Hamilton C. Pólos de crescimento: teoria e política econômica. In: HADDAD, Paulo R. Planejamento regional: métodos e aplicações do caso brasileiro. Rio de Janeiro: IPEA, 1972. 